\title{
Analysis of acoustic emission signals of prestressed reinforced concrete beam in the process of the destruction based on the theory of b-value in Seismology
}

\author{
Fan Xu-hong ${ }^{1, a^{*}}$ \\ ${ }^{1}$ Faculty of civil engineering and mechanics, Jiangsu University, Zhenjiang212013, China
}

Keywords: Prestressed reinforced concrete; Acoustic emission technology; The b-value of Seismology

Abstract: Acoustic emission data of the whole Acoustic emission data of the whole failure process of prestressed reinforced concrete beams by the test of hierarchical loading are collected, then introduce a detailed description of using the least squares method to calculate the b-value using Seismology b-value theory as the basis and study the change of the b-value.The results show:theoretical analysis of acoustic emission data is feasible by using the b-value of seismology,at the same time, there is a certain relationship between the process of the b-value change and the failure process of the specimen.

\section{Introduction}

Different types of cracks generate different types of acoustic emission signals with different frequency ranges. These differences are related to the extent damage of the structure. The micro cracks will produce a large number of small amplitude of acoustic emission events and macro cracks will produce a small number of large amplitude of acoustic emission events. "Seismology b-value analysis method can consider all of these factors, it can be a new way to study and interpret the acoustic emission in the process of monitoring acoustic emission data.

The domestic and foreign scholars have conducted in-depth exploration and research about the theoretical analysis of acoustic emission data by using seismology b-value theory:R.Vidya Sagar ${ }^{[1]}$ study the the change of the $b$-value of prestress reinforced concrete beam under different loading rate through experiment, the results show that the faster the loading rate, the faster the b-value change; By calculation,Aggelis $\mathrm{DG}^{[3]}$ decide the change range of the value of the damage process of concrete; The main research direction of Schumacher $\mathrm{T}^{[4]}$ is the change of the load of the reinforced concrete highway bridge; The acoustic emission data is collected in the loading process are analyzed by using the theory of $b$-value by Colombo $S^{[5]}$; The feasibility of using b-value to analyze the acoustic emission signal in high speed bridge detection is described by Schumacher $\mathrm{T}^{[6]}$

\section{b-value of Seismology and b-value of acoustic emission}

b-value of Seismology

In seismology, the number of large magnitude events is less than small magnitude events. This fact can be quantified as the empirical formula for the magnitude of the relationship between the frequency and the magnitude of the Richter and Guntenberg:

$$
\log _{10} N(M)=a-b M_{L} \text {. }
$$

In this formula,the $M_{L}$ means magnitude of the events, $N(M)$ means the sum of magnitude events greater than $\mathrm{M}, \mathrm{a}$ and $\mathrm{b}$ are the empirical constants.In seismology, $\mathrm{b}$-values are defined as the negative gradient of the relationship between the logarithmic seismic frequency and the magnitude .

$1.2 \mathrm{~b}$-value of Acoustic emission

For the data collected in the acoustic emission test, some scholars use the above formula (1) directly. In fact, collected acoustic emission test amplitude is in DB as a unit, and the definition of Seismology on the Richter scale is the common logarithm of the calculus of maximum amplitude in the 
observation point of seismic instrument recorded by seismic wave. Therefore, the above formula (1) must be developed in order to be used to calculate the data of acoustic emission test.

In conclusion, the empirical formula proposed by Richter and Guntenberg can be deformed when using the acoustic emission technique like this:

$$
\log _{10} N(M)=a-b\left(\frac{A_{d B}}{20}\right)
$$

In this formula: $A_{d B}$ means the amplitude value of the acoustic emission in the $\mathrm{DB}, N$ refers to the growth frequency, $a$ is the empirical constant and $b$ is the $b$-value of the acoustic emission.

1.3 Physical meaning of the $b$-value in acoustic emission

From the above mentioned above things, the b-value can be defined as the negative gradient of the logarithmic relationship between the acoustic emission event frequency and the acoustic emission amplitude. The corresponding relationship between the two parts in the process of loading can be represented by the following figure1:At the early stage of loading, the number of small amplitude events is large, the large amplitude event number is small, so the negative gradient of the logarithmic relationship between the two aspects is fluctuation in the larger step, The fit of the two straight line slope is steeper; When the specimen was beginning to produce macro crack, the number of large amplitude events suddenly began to increase, the proportion of the total number of acoustic emission events is increasing, so that the slope of the fitting curve is slowly.

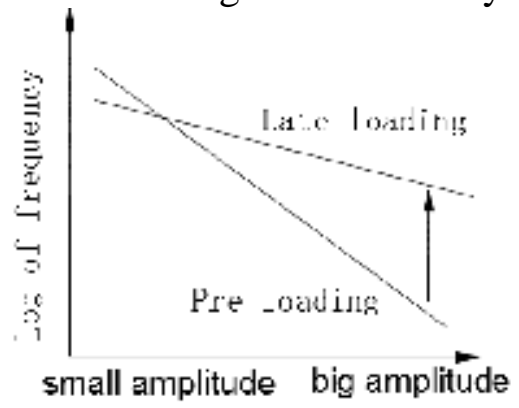

Figure 1 Physical meaning of the b-value of acoustic emission

\section{Test content}

Preparation of specimen

Prestressed reinforced concrete beam is tested, the size is: $150 \mathrm{~mm} \times 250 \mathrm{~mm} \times 1200 \mathrm{~mm}$,concrete grade is $\mathrm{C} 40$ and $\mathrm{C50}$,match ratio is shown as Tab1.According to the optimal reinforcement beam reinforcement,

\begin{tabular}{ccccccc}
\hline Mix proportion & Cement & Sand & Gravel & Water & $\begin{array}{c}\text { Water cement } \\
\text { ratio }\end{array}$ & Water reducing agent \\
\hline$C 40$ & 1 & 2.48 & 4.61 & 0.53 & 0.53 & 0.015 \\
$C 50$ & 1 & 1.06 & 1.94 & 0.3 & 0.3 & 0.03 \\
\hline
\end{tabular}

Tab.1 Mix proportions of concrete

choosing $2^{\phi} 10$ as a reinforcing bar,frame bar is $2^{\phi} 10$,prestressed reinforcement using 1 x 3 strand steel,in the prestressing tendons and the lower part of the reinforced bar, a strain gauge is added to measure the bond stress and relative slip.Embedded metal bellows, support with anchor, in the tensile steel span parts with grinding wheel will be polished with strain gage rib, each piece of tension in the middle part of the strain gauge is attached and there is a total of four strains, leading to the template from the wire and seal with epoxy resin after drying. Maintenance for $24 \mathrm{~h}$ and measure the bond stress and relative slip.

\subsection{Test equipment}

The test instrument is SAEU2S-2 4 channel USB technology from Hua Na acoustic emission system. Loading equipment is manual oil pressure jack of 20 ton, the using the anti force frame can provide vertical load. Through the oil pressure meter reading control size, the manual device can 
effectively avoid the mechanical noise, and improve the acoustic emission signal to noise ratio. Test apparatus is shown in Figure 2.

loading mode

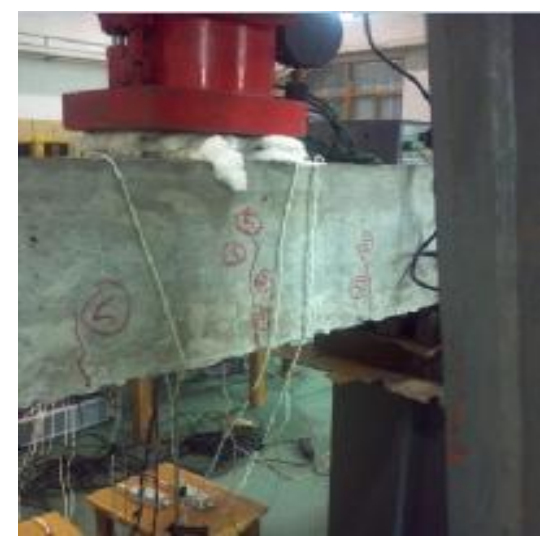

Fig. 2 test loading device

This test is loaded into a hierarchical load mode, which is divided into 10 stages,the ultimate bearing capacity equals to the final value.The i phase of the loading force can also be regarded as the $0.1 \mathrm{i}$ times of the ultimate stress level, each stage of loading as shown in Table 1:

\begin{tabular}{ccc}
\hline Loading stage & $C 40(k N)$ & $C 50(k N)$ \\
\hline 1 & 3.36 & 3.95 \\
2 & 9.4 & 10.96 \\
3 & 15.44 & 17.97 \\
4 & 21.49 & 24.97 \\
5 & 27.53 & 31.98 \\
6 & 33.57 & 38.99 \\
7 & 39.61 & 45.95 \\
8 & 45.65 & 53 \\
9 & 54.34 & 62.33 \\
$10($ Limit $)$ & 63.04 & 71.67 \\
\hline
\end{tabular}

Tab.2 Loading force at each stage

\section{Test results and analysis}

calculation method of b-value

The raw data recorded with the system were processed using Matlab in order to carry out a b-value analysis.During all the processing the first stage was ignored as the numbers of AE events was not significant. First the range of amplitude was decided, going from a threshold of 40dB to a maximum of $100 \mathrm{~dB}$ in steps of $5 \mathrm{~dB}$. Then, the number of acoustic emission events in each stage of loading is grouped according to the amplitude values.At the same time, the linear correlation coefficient of the data is calculated to demonstrate the fitting degree between the two groups of date and the error analysis between the measured value of the data and the fitting value is analyzed 3.2 Test data processing

For the acoustic emission data collected in the experimental process of $\mathrm{C} 40$ beam and C50 beam are used to calculate $b$-value as the above calculation method. From this, the corresponding values of each beam in each loading stage are obtained.

The mean value of each loading stage obtained the channel 2 and channel 3 is plotted as a line graph, as shown in Figure 6 and 7, to facilitate the observation of the change. 

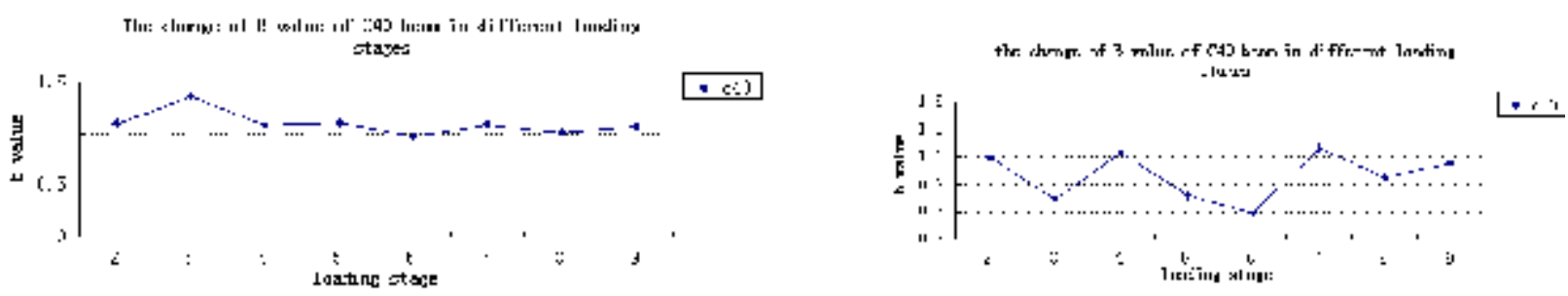

Fig. 3 the change of b-value of C40 and C50 beam in different loading stages

As shown, it can be seen that the b-value of the initial stage of loading is in a trend of fluctuation in a large range, and when it is in the sixth stage, the values are in the lowest position.

\section{Conclusions}

This paper mainly discusses the application of seismology b-value theory in detection of prestressed concrete beam with acoustic emission, now can draw the following conclusions:

(1)After calculation formula transformation of the Seismology b-value can be applied to the field of acoustic emission detection. The b-value of AE events is the linear relationship with the logarithm of the acoustic emission events and the amplitude value, which changes when the two aspects change.

(2)The broken line graph of b-value is calculated by the least square method. The change trend of the method is related to the formation of the cracks in the prestressed concrete beams. At the early time of loading, the prestressed reinforced concrete beam has no marc crack; the b-value has changed at a larger position. When the b-value drops to the lowest point, the middle part of the specimen is beginning to produce the macro crack.

Through the above experimental process and analysis of the process can be found that the change of the b-value has a certain relationship with the process of the formation of the crack.But this paper only analyzes the change of the loading stage, without the analysis of the unloading stage.At the same time, due to the fact that in real life, the stress of prestressed reinforced concrete structures is more complicated, and there are a lot of limiting conditions for the use of acoustic emission technology. This paper makes a qualitative analysis of the relationship between the b-value and the crack of the specimen, but the use of this method for quantitative analysis still needs to make efforts.

\section{References}

[1]R.Vidya Sagar M.V.M.S. Rao An experimental study on loading rate effect on acoustic emission based b-values related to reinforced concrete fracture [J]. Construction and Building Materials. 2012; 42:1094-1104

[2]Alberto Carpinteri From criticality to final collapse: Evolution of the " $b$-value" from 1.5 to $1.0[\mathrm{~J}]$. Chaos, Solitons and Fractals. 2009; 41:843-853

[3]Aggelis DG. Classification of cracking in concrete by acoustic emission parameters[J]. Mech Res Comm.2011; 38(3):153-157

[4]Schumacher T,Higgins C,Lovejoy S. Estimating operating load conditions on reinforced concrete highway bridges with b-value analysis from acoustic emission monitoring[J]. Struct Health Monit 2011; 10(1):17-32

[5]Colombo S, Main IG, Forde MC. Assessing damage of reinforced concrete beam using b-value analysis of acoustic emission signals [J]. ASCE J Mater Civil Eng. 2003; 15(3):280-286

[6]Schumacher T,Higgins CC.Estimating operating load conditions on reinforced concrete highway bridges with $b$-value analysis from acoustic emission monitoring[J]. Struct Health Monitor .2011; 10(1):17-32 
[7]Li Xiaojun, Guangqi, Li Hua Min. Based on AE b-value variation of rock failure precursor recognition and its limitations. Henan University of science and Technology Journal [J]. 2010; 29(5):663-666 